\title{
Covariant map between Ramond-Neveu-Schwarz and pure spinor formalisms for the superstring
}

\author{
Nathan Berkovits \\ ICTP South American Institute for Fundamental Research Instituto de Física Teórica, \\ UNESP - Univ. Estadual Paulista \\ Rua Dr. Bento T. Ferraz 271, 01140-070, São Paulo, SP, Brasil \\ E-mail: nberkovi@ift.unesp.br
}

ABSTRACT: A covariant map between the Ramond-Neveu-Schwarz (RNS) and pure spinor formalisms for the superstring is found which transforms the RNS and pure spinor BRST operators into each other. The key ingredient is a dynamical twisting of the ten spin-half RNS fermions into five spin-one and five spin-zero fermions using bosonic pure spinors that parameterize an $\mathrm{SO}(10) / \mathrm{U}(5)$ coset. The map relates massless vertex operators in the two formalisms, and gives a new description of Ramond states which does not require spin fields. An argument is proposed for relating the amplitude prescriptions in the two formalisms.

Keywords: Superstrings and Heterotic Strings, Topological Strings

ARXIV EPRINT: 1312.0845 


\section{Contents}

1 Introduction $\quad 1$

2 Covariant map $\quad 4$

2.1 Non-minimal RNS formalism 4

2.2 Dynamical twisting 6

3 Vertex operators $\quad 10$

$\begin{array}{lll}3.1 & \text { Non-minimal RNS vertex operators } & 10\end{array}$

$\begin{array}{lll}3.2 & \text { Relation with RNS and pure spinor vertex operators } & 12\end{array}$

4 Scattering amplitudes $\quad 13$

$\begin{array}{lll}4.1 & \text { Non-minimal RNS amplitude prescription } & 13\end{array}$

$\begin{array}{lll}4.2 & \text { Pure spinor amplitude prescription } & 14\end{array}$

\section{Introduction}

Although the Ramond-Neveu-Schwarz (RNS) formalism for the superstring has an elegant worldsheet description as an $\mathrm{N}=1$ superconformal field theory, its spacetime description is complicated. Vertex operators for Ramond states require spin fields and it is unknown how to describe the RNS formalism in Ramond-Ramond backgrounds. Furthermore, the RNS scattering amplitude prescription requires summing over spin structures to project out states in the $G S O(-)$ sector, and cancellations implied by spacetime supersymmetry are far from manifest.

On the other hand, the pure spinor formalism for the superstring has an elegant spacetime description in which vertex operators are expressed in $d=10$ superspace and spacetime supersymmetry is manifest. However, its worldsheet description is mysterious and the pure spinor BRST operator has not yet been derived from gauge-fixing a worldsheet reparamaterization-invariant action.

Constructing a map between these two superstring formalisms is an obvious way to better understand both formalisms. In light-cone gauge, the pure spinor formalism is equivalent to the light-cone Green-Schwarz (GS) formalism which was mapped in [1] to light-cone RNS. This map manifestly preserves an $\mathrm{SU}(4)$ subgroup of the $\mathrm{SO}(8)$ light-cone symmetry and transforms the eight light-cone RNS vector variables $\psi^{j}$ into the eight lightcone GS spinor variables $\theta^{a}$. Splitting the $\mathrm{SO}(8)$ vector $\psi^{j}$ and $\mathrm{SO}(8)$ spinor $\theta^{a}$ as $\left(\psi^{J}, \psi_{J}\right)$ and $\left(\theta^{J}, \theta_{J}\right)$ where $J=1$ to 4 is an $\mathrm{SU}(4)$ index, the map of [1] is obtained by bosonizing

$$
\psi^{J}=e^{i \sigma_{J}}, \quad \psi_{J}=e^{-i \sigma_{J}}
$$


and writing $\left(\theta^{J}, \theta_{J}\right)$ as the spin fields

$$
\theta^{J}=e^{i \sigma_{J}-\frac{i}{2}\left(\sigma_{1}+\sigma_{2}+\sigma_{3}+\sigma_{4}\right)}, \quad \theta_{J}=e^{-i \sigma_{J}+\frac{i}{2}\left(\sigma_{1}+\sigma_{2}+\sigma_{3}+\sigma_{4}\right)} .
$$

Note that all $\left(\psi^{J}, \psi_{J}\right)$ and $\left(\theta^{J}, \theta_{J}\right)$ variables carry conformal weight $\frac{1}{2}$.

To find a covariant version of this map, the first step is to enlarge the SU(4) symmetry of (1.1) to an $\mathrm{SU}(5)$ subgroup of the (Wick-rotated) $\mathrm{SO}(10)$ Lorentz group. In the "U(5) hybrid formalism" of [2], this was done by splitting the RNS SO(10) vector $\psi^{m}$ for $m=0$ to 9 into $\left(\psi^{A}, \psi_{A}\right)$ where $A=1$ to 5 is an $\mathrm{SU}(5)$ index, bosonizing as

$$
\psi^{A}=e^{i \sigma_{A}}, \quad \psi_{A}=e^{-i \sigma_{A}},
$$

and constructing 5 of the 16 components of the $\mathrm{SO}(10)$ spinor $\theta^{\alpha}$ and its conjugate momentum $p_{\alpha}$ as the spin fields

$$
\theta^{A}=e^{i \sigma_{A}-\frac{i}{2}\left(\sigma_{1}+\sigma_{2}+\sigma_{3}+\sigma_{4}+\sigma_{5}\right)} e^{\frac{1}{2} \phi}, \quad p_{A}=e^{-i \sigma_{A}+\frac{i}{2}\left(\sigma_{1}+\sigma_{2}+\sigma_{3}+\sigma_{4}+\sigma_{5}\right)} e^{-\frac{1}{2} \phi}
$$

where $\phi$ comes from the Friedan-Martinec-Shenker bosonization [3] of the $(\beta, \gamma)$ ghosts as $\beta \gamma=\partial \phi$. Note that $\theta^{A}$ carries conformal weight zero as in the covariant GS formalism and its conjugate momentum $p_{A}$ carries conformal weight +1 . However, the other 11 components of the $\mathrm{SO}(10)$ spinors $\theta^{\alpha}$ and $p_{\alpha}$ were absent in this $\mathrm{U}(5)$ hybrid formalism so $\mathrm{SO}(10)$ covariance was not manifest.

In the pure spinor formalism for the superstring, all 16 components of $\theta^{\alpha}$ and $p_{\alpha}$ are present as well as the 11 independent components of a bosonic pure spinor $\lambda^{\alpha}$ satisfying $\lambda \gamma^{m} \lambda=0$ and its conjugate momentum $w_{\alpha}$. A map was proposed in [4] between the RNS and pure spinor formalism which combined the $\mathrm{U}(5)$ hybrid formalism with a "topological" sector containing $\left(\lambda^{\alpha}, w_{\alpha}\right)$ and the 11 remaining components of $\left(\theta^{\alpha}, p_{\alpha}\right)$. However, because of the complicated bosonization formula of (1.3) used in the hybrid formalism, the map was not manifestly $\mathrm{SO}(10)$ covariant. Although there exists a relation at the classical level between the hybrid formalism and the manifestly covariant "superembedding" formalisms [5-7], this relation has not yet been understood at the quantum level.

In [8], a new approach to relating the RNS and pure spinor variables was proposed in which bosonization of the RNS ghost and matter fields is unnecessary. In this approach, one simply rescales the $\mathrm{U}(5)$ components $\left(\psi^{A}, \psi_{A}\right)$ of $\psi^{m}$ in opposite directions using the $\gamma$ ghost as

$$
\Gamma^{A}=\gamma \psi^{A}, \quad \bar{\Gamma}_{A}=\frac{1}{\gamma} \psi_{A},
$$

where $\Gamma^{A}$ and $\bar{\Gamma}_{A}$ are GSO-even fermions of conformal weight 0 and 1 . This "twisting" by the $\gamma$ ghost was earlier used in the Calabi-Yau fermions of the $\mathrm{d}=4$ hybrid formalism [9] and also appeared in the topological twisting papers of Baulieu et al. [10-13]. Although (1.4) breaks $\mathrm{SO}(10)$ covariance to $\mathrm{U}(5)$, one can recover the full $\mathrm{SO}(10)$ covariance by using the pure spinor $\lambda^{\alpha}$ and its complex conjugate $\bar{\lambda}_{\alpha}$ to "dynamically" choose the $\mathrm{U}(5)$ direction of the twisting so that

$$
\Gamma^{m}=\gamma \frac{\lambda \gamma^{m} \gamma^{n} \bar{\lambda}}{2(\lambda \bar{\lambda})} \psi_{n}, \quad \bar{\Gamma}_{m}=\frac{1}{\gamma} \frac{\lambda \gamma_{n} \gamma_{m} \bar{\lambda}}{2(\lambda \bar{\lambda})} \psi^{n}
$$


Note that $\Gamma^{m}$ and $\bar{\Gamma}_{m}$ each have five independent components since they satisfy $\Gamma^{m}\left(\gamma_{m} \lambda\right)_{\alpha}=$ $\bar{\Gamma}_{m}\left(\gamma^{m} \bar{\lambda}\right)^{\alpha}=0$, and were related in [8] to five components of $\theta^{\alpha}$ and $p_{\alpha}$.

Although both the bosonization formulas of (1.3) and the twisting formulas of (1.4) and (1.5) map RNS spin-half fermions into GS-like spin-zero and spin-one fermions, the relation of the two maps is unclear. As stressed by Witten [14], bosonization formulas such as (1.3) can be ill-defined at higher genus, but this does not seem to be a problem for the twisting formulas of (1.4) and (1.5). The rigid twisting of (1.4) is related to holomorphic $\mathrm{d}=5$ super-Yang-Mills $[15,16]$, and it was conjectured by Nekrasov in [15] that the dynamical twisting of (1.5) replaces the topogical spectrum of holomorphic $d=5$ superYang-Mills with the $\mathrm{d}=10$ superstring spectrum. Evidence for Nekrasov's conjecture was obtained recently in [17] where the "dynamical twisting" of (1.5) was shown to simplify the expression for the composite $b$ ghost in the pure spinor formalism. And in this paper, Nekrasov's conjecture will be confirmed by showing that it maps the RNS and pure spinor BRST operators into each other.

To use the dynamical twisting procedure of (1.5) to provide a covariant map between the RNS and pure spinor BRST operators, the first step will be to add to the usual RNS variables a topological set of "non-minimal" fermionic and bosonic spacetime spinor variables $\left(\theta^{\alpha}, p_{\alpha}\right)$ and $\left(\Lambda^{\alpha}, \Omega_{\alpha}\right)$ of conformal weight $(0,1)$. The BRST operator in this "non-minimal" RNS formalism will be defined as

$$
Q=Q_{\mathrm{RNS}}+\int d z\left(\Lambda^{\alpha} p_{\alpha}\right)
$$

so that the cohomology of physical states is unchanged. After the similarity transformation $Q \rightarrow e^{-R} Q e^{R}$ where

$$
R=\frac{1}{2 \gamma}\left(\Lambda \gamma_{m} \theta\right) \psi^{m}
$$

the non-minimal BRST operator of (1.6) can be surprisingly written in manifestly spacetime supersymmetric form where $\left(x^{m}, \theta^{\alpha}\right)$ transform as $\mathrm{d}=10$ superspace variables. Furthermore, despite the presence of $\frac{1}{\gamma}$ in (1.7), vertex operators in the $G S O(+)$ sector can be written in $\mathrm{d}=10$ superspace and do not contain any inverse powers of $\gamma$. So Ramond states in this non-minimal RNS formalism do not require spin fields or bosonization and one can easily describe the formalism in curved Ramond-Ramond backgrounds.

To perform dynamical twisting as in (1.5), one decomposes the unconstrained bosonic spinor $\Lambda^{\alpha}$ into pure spinors $\lambda^{\alpha}$ and $\bar{\lambda}_{\alpha}$ by defining $[18,19]$

$$
\Lambda^{\alpha}=\lambda^{\alpha}+\frac{1}{2(\lambda \bar{\lambda})} u^{m}\left(\gamma_{m} \bar{\lambda}\right)^{\alpha}
$$

where $u^{m}$ is a bosonic vector with only five independent components because of the gauge invariance $\delta u^{m}=\left(\epsilon \gamma^{m} \bar{\lambda}\right)$. The definition of (1.8) for the unconstrained $\Lambda^{\alpha}$ might be useful for understanding the relation with "extended" versions of the pure spinor formalism such as [20-23] in which the spinor ghosts were unconstrained. After defining $\Gamma^{m}$ and $\bar{\Gamma}_{m}$ as in (1.5), the RNS $\gamma$ ghost only appears in even powers so it is convenient to define a new ghost variable $\widehat{\gamma} \equiv(\gamma)^{2}$. Since $\widehat{\gamma}$ and its conjugate momentum $\widehat{\beta}$ carry conformal weight -1 and +2 , the contribution of $\left(\Gamma^{m}, \bar{\Gamma}_{m}\right)$ and $(\widehat{\beta}, \widehat{\gamma})$ to the conformal anomaly is $-10+26$ which is equal to the $+5+11$ contribution of the original $\psi^{m}$ and $(\beta, \gamma)$ variables. 
Expressing the non-minimal RNS BRST operator in terms of $\left(\Gamma^{m}, \bar{\Gamma}_{m}\right)$ and $(\widehat{\beta}, \widehat{\gamma})$ and decomposing $\Lambda^{\alpha}$ as in (1.8), one finds after a similarity transformation that

$$
Q=\int d z\left(\lambda^{\alpha} d_{\alpha}+\bar{w}^{\alpha} r_{\alpha}+u^{m} \bar{\Gamma}_{m}+\widehat{\gamma} b\right)
$$

where $\int d z\left(\lambda^{\alpha} d_{\alpha}\right)$ is the pure spinor BRST operator. So after adding non-minimal spinor variables to the RNS formalism, dynamically twisting as in (1.5), and performing various similarity transformations, the RNS BRST operator is covariantly mapped into the pure spinor BRST operator plus a set of "topological" variables which decouple from the cohomology. Furthermore, the non-minimal RNS formalism containing both the RNS $\psi^{m}$ variables and the GS $\theta^{\alpha}$ variables provides a natural bridge between the RNS and pure spinor formalisms which resembles the "superembedding" formalisms reviewed in [7]. Vertex operators in the $G S O(+)$ sector can be expressed in $\mathrm{d}=10$ superspace using the non-minimal RNS formalism, and in the gauge $u^{m}=\Gamma^{m}=0$, they reduce to the usual pure spinor vertex operators. And in the gauge $\Lambda^{\alpha}=\theta^{\alpha}=0$, the vertex operators for bosons in the non-minimal RNS formalism reduce to the usual Neveu-Schwarz vertex operators of the RNS formalism in the zero picture.

The covariant map can also be used to relate the scattering amplitude prescriptions in the RNS and pure spinor formalisms. Both of these formalisms contain chiral bosons, and functional integration over chiral bosons is divergent because of their non-compact zero modes. These divergences are cancelled by zeros coming from functional integration over the zero modes of chiral fermions, and a convenient BRST-invariant method for regularizing the divergence is to insert a picture-changing operator for each chiral boson zero mode. Since the dynamical twisting procedure changes the $(\beta, \gamma)$ chiral bosons of the RNS formalism to $(\widehat{\beta}, \widehat{\gamma})$ chiral bosons which carry different conformal weight, the number and type of picture-changing operators inserted in the RNS and pure spinor formalism are different. Nevertheless, assuming that the dynamical twisting procedure is a consistent field redefinition at the quantum level, one expects that the different RNS and pure spinor prescriptions for regularizing the chiral boson zero modes should lead to the same scattering amplitude.

In section 2 of this paper, the non-minimal RNS formalism is constructed and the dynamical twisting procedure is defined which covariantly maps the RNS BRST operator into the pure spinor BRST operator. In section 3, the massless vertex operators in the non-minimal RNS formalism are constructed and shown to form a bridge between the RNS and pure spinor vertex operators. And in section 4, the RNS and pure spinor scattering amplitude prescriptions are related to each other through the dynamical twisting procedure.

\section{Covariant map}

\section{$2.1 \quad$ Non-minimal RNS formalism}

The usual RNS worldsheet action, stress tensor, and BRST operator are

$$
S_{\mathrm{RNS}}=\int d^{2} z\left(\frac{1}{2} \partial x^{m} \bar{\partial} x_{m}+\frac{1}{2} \psi^{m} \bar{\partial} \psi_{m}+\beta \bar{\partial} \gamma+b \bar{\partial} c\right)
$$




$$
\begin{aligned}
T_{\mathrm{RNS}} & =-\frac{1}{2} \partial x^{m} \partial x_{m}-\frac{1}{2} \psi^{m} \partial \psi_{m}-\beta \partial \gamma-\frac{1}{2} \partial(\beta \gamma)-b \partial c-\partial(b c), \\
Q_{\mathrm{RNS}} & =\int d z\left(c T_{\mathrm{RNS}}+\gamma \psi^{m} \partial x_{m}+\gamma^{2} b-b c \partial c\right)
\end{aligned}
$$

where the right-moving variables $\left(\bar{\psi}^{m}, \bar{\beta}, \bar{\gamma}, \bar{b}, \bar{c}\right)$ will be ignored throughout this paper. The free field OPE's of the left-moving RNS variables of (2.1) are

$$
\begin{aligned}
\partial x^{m}(z) \partial x^{n}(0) & \rightarrow-z^{-2} \eta^{m n}, & \psi^{m}(z) \psi^{n}(0) & \rightarrow z^{-1} \eta^{m n}, \\
\gamma(z) \beta(0) & \rightarrow z^{-1}, & c(z) b(0) & \rightarrow z^{-1} .
\end{aligned}
$$

Although only the open superstring will be discussed in this paper, all results can be easily generalized to the closed superstring by taking the "left-right product" of two open superstrings.

To relate (2.1), (2.2) and (2.3) to the pure spinor worldsheet action, stress tensor, and BRST operator, the first step is to add a non-minimal set of fermionic spacetime spinor variables $\left(\theta^{\alpha}, p_{\alpha}\right)$ of conformal weight $(0,1)$ and bosonic unconstrained spacetime spinor variables $\left(\Lambda^{\alpha}, \Omega_{\alpha}\right)$ of conformal weight $(0,1)$ so that

$$
\begin{aligned}
& S=S_{\mathrm{RNS}}+\int d^{2} z\left(p_{\alpha} \bar{\partial} \theta^{\alpha}+\Omega_{\alpha} \bar{\partial} \Lambda^{\alpha}\right) \\
& T=T_{\mathrm{RNS}}-p_{\alpha} \partial \theta^{\alpha}-\Omega_{\alpha} \partial \Lambda^{\alpha} \\
& Q=Q_{\mathrm{RNS}}+\int d z\left(\Lambda^{\alpha} p_{\alpha}\right)
\end{aligned}
$$

with the free field OPE's

$$
\Lambda^{\alpha}(z) \Omega_{\beta}(0) \rightarrow z^{-1} \delta_{\beta}^{\alpha}, \quad \theta^{\alpha}(z) p_{\beta}(0) \rightarrow z^{-1} \delta_{\beta}^{\alpha}
$$

Using the usual quartet argument, the BRST cohomology is unchanged. Performing the similarity transformation $\mathcal{O} \rightarrow e^{-R} \mathcal{O} e^{R}$ on all operators $\mathcal{O}$ where

$$
R=\int d z c \Omega_{\alpha} \partial \theta^{\alpha}
$$

the BRST operator of (2.7) is transformed into the more conventional form

$$
Q=\int d z\left(\Lambda^{\alpha} p_{\alpha}+c T+\gamma \psi^{m} \partial x_{m}+\gamma^{2}\left(b+\Omega_{\alpha} \partial \theta^{\alpha}\right)-b c \partial c\right)
$$

where $T$ is defined in (2.6).

Since the worldsheet variables include the $\left(\theta^{\alpha}, p_{\alpha}\right)$ variables of $\mathrm{d}=10$ superspace, one can construct the operators [24]

$$
q_{\alpha}=\int d z\left(p_{\alpha}+\frac{1}{2}\left(\partial x^{m}+\frac{1}{12} \theta \gamma^{m} \partial \theta\right)\left(\gamma_{m} \theta\right)_{\alpha}\right)
$$


which generate the $\mathrm{d}=10$ spacetime supersymmetry algebra $\left\{q_{\alpha}, q_{\beta}\right\}=\gamma_{\alpha \beta}^{m} \int d z \partial x_{m}$. Although $q_{\alpha}$ does not anticommute with the BRST operator of (2.10), one can perform the further similarity transformation $\mathcal{O} \rightarrow e^{-R^{\prime}} \mathcal{O} e^{R^{\prime}}$ where

$$
R^{\prime}=\int d z \frac{1}{2 \gamma}\left(\Lambda \gamma^{m} \theta\right) \psi_{m}
$$

under which the BRST operator of (2.10) transforms into the manifestly spacetime supersymmetric operator

$$
Q=\int d z\left(\Lambda^{\alpha} d_{\alpha}+\frac{1}{2 \gamma}\left(\Lambda \gamma^{m} \Lambda\right) \psi_{m}+c T+\gamma \psi^{m} \Pi_{m}+\gamma^{2}\left(b+\Omega_{\alpha} \partial \theta^{\alpha}\right)-b c \partial c\right)
$$

where

$$
d_{\alpha}=p_{\alpha}-\frac{1}{2}\left(\partial x^{m}+\frac{1}{4} \theta \gamma^{m} \partial \theta\right)\left(\gamma_{m} \theta\right)_{\alpha}, \quad \Pi^{m}=\partial x^{m}+\frac{1}{2} \theta \gamma^{m} \partial \theta
$$

are the usual spacetime supersymmetric operators [24] for fermionic and bosonic momenta. Note that $T$ of (2.6) can be written in the manifestly spacetime supersymmetric form

$$
T=-\frac{1}{2} \Pi^{m} \Pi_{m}-d_{\alpha} \partial \theta^{\alpha}-\Omega_{\alpha} \partial \Lambda^{\alpha}-\frac{1}{2} \psi^{m} \partial \psi_{m}-\beta \partial \gamma-\frac{1}{2} \partial(\beta \gamma)-b \partial c-\partial(b c) .
$$

So after adding the non-minimal spinor variables and performing the similarity transformation of (2.12), the non-minimal RNS BRST operator and stress tensor of (2.13) and (2.15) are manifestly invariant under the spacetime supersymmetry generated by (2.11). But because of the inverse power of $\gamma$ in the similarity transformation of (2.12) and in the term $\frac{1}{2 \gamma}\left(\Lambda \gamma^{m} \Lambda\right) \psi_{m}$ in the BRST current, the Hilbert space of states in the non-minimal RNS formalism is no longer the usual "small" Hilbert space of the RNS formalism in which all states are polynomials in $\beta$ and $\gamma$. Nevertheless, it will be shown in section 3 that all states in the $G S O(+)$ sector of the non-minimal RNS formalism can be described in the "small" Hilbert space and that spacetime supersymmetry acts covariantly on these states. Furthermore, it will now be shown that after twisting the ten spin-half variables $\psi^{m}$ into five spin-zero and five spin-one variables, the inverse powers of $\gamma$ can be eliminated from the BRST operator and the resulting twisted version of the non-minimal RNS formalism is the pure spinor formalism.

\section{$2.2 \quad$ Dynamical twisting}

To covariantly twist the ten $\psi^{m}$ spin-half variables into five spin-zero and five spin-one variables, one needs to construct pure spinor variables $\left(\lambda^{\alpha}, \bar{\lambda}_{\alpha}\right)$ satisfying the constraints

$$
\lambda \gamma^{m} \lambda=0, \quad \bar{\lambda} \gamma^{m} \bar{\lambda}=0,
$$

whose 11 complex components (in Wick-rotated Euclidean space) parameterize the complex space $\frac{\mathrm{SO}(10)}{\mathrm{U}(5)} \times C$. In terms of the unconstrained spinor $\Lambda^{\alpha}, \lambda^{\alpha}$ and $\bar{\lambda}_{\alpha}$ will be defined as $[18,19]$

$$
\Lambda^{\alpha}=\lambda^{\alpha}+\frac{1}{2(\lambda \bar{\lambda})} u^{m}\left(\gamma_{m} \bar{\lambda}\right)^{\alpha}
$$


where $u^{m}$ is a bosonic vector defined up to the gauge transformation

$$
\delta u^{m}=\epsilon_{\alpha}\left(\gamma^{m} \bar{\lambda}\right)^{\alpha} .
$$

Note that $\Lambda^{\alpha}$ in (2.17) is unchanged under the gauge transformation

$$
\delta \bar{\lambda}_{\alpha}=\xi_{\alpha}, \quad \delta u^{m}=-\frac{1}{2(\lambda \bar{\lambda})}\left(\lambda \gamma^{m} \gamma^{n} \xi\right) u_{n}, \quad \delta \lambda^{\alpha}=\frac{1}{16(\lambda \bar{\lambda})^{2}}\left(\bar{\lambda} \gamma^{m n} \gamma^{p} \xi\right)\left(\gamma_{m n} \lambda\right)^{\alpha} u_{p},
$$

where $\xi_{\alpha}$ is any spinor satisfying $\bar{\lambda} \gamma^{m} \xi=0$. The gauge transformations of (2.18) and (2.19) can be used to gauge-fix all 11 components of $\bar{\lambda}_{\alpha}$ and 5 components of $u^{m}$, and the remaining 16 components of $u^{m}$ and $\lambda^{\alpha}$ are determined by $\Lambda^{\alpha}$.

Defining $w_{\alpha}$ and $v_{m}$ to be the conjugate momenta to $\lambda^{\alpha}$ and $u_{m}$, one finds that

$$
\Omega_{\alpha}=\frac{1}{4(\lambda \bar{\lambda})}\left[\left(\bar{\lambda} \gamma^{m n}\right)_{\alpha} N_{m n}+\bar{\lambda}_{\alpha}\left(J+4 u_{m} v^{m}\right)\right]+\left(\lambda \gamma^{m}\right)_{\alpha} v_{m}
$$

satisfies the desired OPE $\Lambda^{\alpha}(z) \Omega_{\beta}(0) \rightarrow z^{-1} \delta_{\beta}^{\alpha}$ where

$$
N_{m n}=\frac{1}{2}\left(\lambda \gamma_{m n} w\right), \quad J=-\lambda^{\alpha} w_{\alpha}
$$

Note that the gauge invariance of (2.18) implies that $v^{m}$ is constrained to satisfy

$$
v^{m}\left(\gamma_{m} \bar{\lambda}\right)^{\alpha}=0
$$

To include the new variables in the formalism, first add $\left(\bar{\lambda}_{\alpha}, \widehat{\bar{w}}^{\alpha}\right)$ and $\left(r_{\alpha}, s^{\alpha}\right)$ to $(2.5),(2.6)$ and (2.13) as

$$
\begin{aligned}
S= & S_{\mathrm{RNS}}+\int d^{2} z\left(p_{\alpha} \bar{\partial} \theta^{\alpha}+\Omega_{\alpha} \bar{\partial} \Lambda^{\alpha}+\widehat{\widehat{w}}^{\alpha} \overline{\partial \lambda}_{\alpha}+s^{\alpha} \bar{\partial} r_{\alpha}\right), \\
T= & T_{\mathrm{RNS}}-p_{\alpha} \partial \theta^{\alpha}-\Omega_{\alpha} \partial \Lambda^{\alpha}-\widehat{\bar{w}}^{\alpha} \partial \bar{\lambda}_{\alpha}-s^{\alpha} \partial r_{\alpha}, \\
Q= & \int d z\left(\Lambda^{\alpha} d_{\alpha}+\frac{1}{2 \gamma}\left(\Lambda \gamma^{m} \Lambda\right) \psi_{m}+c T+\gamma \psi^{m} \Pi_{m}+\gamma^{2}\left(b+\Omega_{\alpha} \partial \theta^{\alpha}\right)-b c \partial c\right) \\
& +\int d z\left(\widehat{\bar{w}}^{\alpha} r_{\alpha}+\gamma^{2} s^{\alpha} \partial \bar{\lambda}_{\alpha}\right)
\end{aligned}
$$

where $\widehat{\bar{w}}^{\alpha}$ has no singular OPE's with $\Lambda^{\alpha}$ or $\Omega_{\alpha}$, and $r_{\alpha}$ is the fermionic ghost coming from the gauge parameter $\xi_{\alpha}$ of (2.19) which is constrained to satisfy

$$
r \gamma^{m} \bar{\lambda}=0
$$

One can then plug into (2.22) and (2.23) the expressions of (2.17) and (2.20) for $\Lambda^{\alpha}$ and $\Omega_{\alpha}$ to find that

$$
\begin{aligned}
& S=S_{\mathrm{RNS}}+\int d^{2} z\left(p_{\alpha} \bar{\partial} \theta^{\alpha}+w_{\alpha} \bar{\partial} \lambda^{\alpha}+v_{m} \bar{\partial} u^{m}+\bar{w}^{\alpha} \overline{\partial \lambda}_{\alpha}+s^{\alpha} \bar{\partial} r_{\alpha}\right), \\
& T=T_{\mathrm{RNS}}-p_{\alpha} \partial \theta^{\alpha}-w_{\alpha} \partial \lambda^{\alpha}-v_{m} \partial u^{m}-\bar{w}^{\alpha} \partial \bar{\lambda}_{\alpha}-s^{\alpha} \partial r_{\alpha},
\end{aligned}
$$


where $\bar{w}^{\alpha}$ has no singular OPE's with $\lambda^{\alpha}$ or $u_{m}$ and is defined by

$$
\bar{w}^{\alpha}=\widehat{\bar{w}}^{\alpha}+\frac{1}{4(\lambda \bar{\lambda})^{2}} u^{n}\left[\left(\lambda \gamma_{m} \gamma_{n} \bar{\lambda}\right)\left(\gamma^{m} w\right)^{\alpha}-2\left(w \gamma_{n} \bar{\lambda}\right) \lambda^{\alpha}-2(\lambda \bar{\lambda}) v^{m}\left(\gamma_{m} \gamma_{n} \lambda\right)^{\alpha}\right] .
$$

Finally, the BRST operator can be expressed in terms of the pure spinor variables and their conjugate momenta by plugging into (2.24) the expressions of $(2.17),(2.20)$, and (2.28) for $\Lambda^{\alpha}, \Omega_{\alpha}$ and $\widehat{\bar{w}}^{\alpha}$ to obtain

$$
\begin{aligned}
Q= & \int d z\left(\lambda^{\alpha} d_{\alpha}+\bar{w}^{\alpha} r_{\alpha}+\gamma \psi^{m} \Pi_{m}+c T-b c \partial c\right. \\
& +\widehat{\gamma}\left[b+s^{\alpha} \partial \bar{\lambda}_{\alpha}+\frac{1}{4(\lambda \bar{\lambda})}\left(\left(\bar{\lambda} \gamma^{m n} \partial \theta\right) N_{m n}+(\bar{\lambda} \partial \theta)\left(J+4 u^{n} v_{n}\right)\right)+\left(\lambda \gamma^{m} \partial \theta\right) v_{m}\right] \\
& \left.+u_{m}\left[\frac{1}{2 \gamma(\lambda \bar{\lambda})}\left(\bar{\lambda} \gamma^{m} \gamma^{n} \lambda\right) \psi_{n}+\frac{1}{2(\lambda \bar{\lambda})} \bar{\lambda} \gamma^{m} d-\frac{\left(\bar{\lambda} \gamma^{m} \gamma^{n p} r\right)}{8(\lambda \bar{\lambda})^{2}} N_{n p}+\frac{\left(\lambda \gamma^{m} \gamma^{n} r\right)}{2(\lambda \bar{\lambda})} v_{n}\right]\right) .
\end{aligned}
$$

Using the pure spinor variables $\left(\lambda^{\alpha}, \bar{\lambda}_{\alpha}\right)$ to covariantly choose the direction of the twisting, one can now dynamically twist the ten spin-half $\psi^{m}$ variables to spin-zero $\Gamma^{m}$ variables and spin-one $\bar{\Gamma}^{m}$ variables defined by

$$
\Gamma^{m}=\frac{1}{2(\lambda \bar{\lambda})} \gamma\left(\lambda \gamma^{m} \gamma^{n} \bar{\lambda}\right) \psi_{n}, \quad \bar{\Gamma}^{m}=\frac{1}{2(\lambda \bar{\lambda})} \frac{1}{\gamma}\left(\bar{\lambda} \gamma^{m} \gamma^{n} \lambda\right) \psi_{n}
$$

so that

$$
\psi^{m}=\gamma \bar{\Gamma}^{m}+\frac{1}{\gamma} \frac{\left(\lambda \gamma^{m} \gamma^{n} \bar{\lambda}\right)}{2(\lambda \bar{\lambda})} \Gamma_{n}
$$

$\bar{\Gamma}^{m}$ will be constrained to satisfy

$$
\bar{\Gamma}^{m}\left(\gamma_{m} \bar{\lambda}\right)^{\alpha}=0
$$

and since $\psi^{m}$ of (2.31) is invariant under the gauge transformation $\delta \Gamma_{m}=\epsilon \gamma_{m} \bar{\lambda}$ generated by (2.32), only half of the $\Gamma^{m}$ and $\bar{\Gamma}^{m}$ components are independent. After performing this twisting and expressing $\psi^{m}$ in terms of $\Gamma^{m}$ and $\bar{\Gamma}^{m}, G S O(+)$ states only depend on even powers of the $\gamma$ ghost. So it will be useful to define

$$
\widehat{\gamma} \equiv(\gamma)^{2}
$$

which carries conformal weight -1 , and define $\widehat{\beta}$ of conformal weight +2 to be the conjugate momentum to $\widehat{\gamma}$.

In terms of $\left(\Gamma^{m}, \bar{\Gamma}_{m}, \widehat{\gamma}, \widehat{\beta}\right)$, the worldsheet action and stress tensor are

$$
\begin{aligned}
S= & \int d^{2} z\left(\frac{1}{2} \partial x^{m} \bar{\partial} x_{m}+\bar{\Gamma}^{m} \bar{\partial} \Gamma_{m}+\widehat{\beta} \partial \widehat{\gamma}+b \bar{\partial} c\right. \\
& \left.+p_{\alpha} \bar{\partial} \theta^{\alpha}+v^{m} \bar{\partial} u_{m}+w_{\alpha}^{\prime} \bar{\partial} \lambda^{\alpha}+\bar{w}^{\prime} \overline{\partial \lambda}_{\alpha}+s^{\alpha} \bar{\partial} r_{\alpha}\right), \\
T= & -\frac{1}{2} \partial x^{m} \partial x_{m}-\bar{\Gamma}^{m} \partial \Gamma_{m}-\widehat{\beta} \partial \widehat{\gamma}-\partial(\widehat{\beta} \widehat{\gamma})-b \partial c-\partial(b c) \\
& -p_{\alpha} \partial \theta^{\alpha}-v^{m} \partial u_{m}-w_{\alpha}^{\prime} \partial \lambda^{\alpha}-\bar{w}^{\alpha} \partial \bar{\lambda}_{\alpha}-s^{\alpha} \partial r_{\alpha},
\end{aligned}
$$


where

$$
\begin{aligned}
\widehat{\beta} & =\frac{1}{2 \gamma} \beta+\frac{1}{2 \gamma^{2}} \Gamma^{m} \bar{\Gamma}_{m}, \\
w_{\alpha}^{\prime} & =w_{\alpha}-\frac{1}{4 \gamma^{2}(\lambda \bar{\lambda})} \Gamma^{m} \Gamma^{n}\left[\left(\bar{\lambda} \gamma_{m n}\right)_{\alpha}+\frac{\left(\lambda \gamma_{m n} \bar{\lambda}\right)}{(\lambda \bar{\lambda})} \bar{\lambda}_{\alpha}\right], \\
\bar{w}^{\prime} \alpha & =\bar{w}^{\alpha}-\frac{\gamma^{2}}{4(\lambda \bar{\lambda})} \bar{\Gamma}^{m} \bar{\Gamma}^{n}\left(\lambda \gamma_{m n}\right)^{\alpha}+\frac{1}{2(\lambda \bar{\lambda})} \Gamma^{m} \bar{\Gamma}^{n}\left(\lambda \gamma_{m} \gamma_{n}\right)^{\alpha},
\end{aligned}
$$

and the conjugate momenta $\widehat{\beta}, w_{\alpha}^{\prime}$ and $\bar{w}^{\prime} \alpha$ of (2.36) have been defined to have no singular OPE's with $\Gamma^{m}$ and $\bar{\Gamma}_{m}$. Note that the twisting of the $\psi^{m}$ variables to $\left(\Gamma^{m}, \bar{\Gamma}_{m}\right)$ variables shifts their central charge contribution from +5 to -10 , and is compensated by the replacement of the $(\beta, \gamma)$ with $(\widehat{\beta}, \widehat{\gamma})$ variables which shifts their central charge contribution from +11 to +26 .

Expressing $Q$ of (2.29) in terms of the twisted variables of (2.30) and (2.33), one obtains

$$
\begin{aligned}
Q= & \int d z\left(\lambda^{\alpha} d_{\alpha}+\widetilde{\widetilde{w}}^{\alpha}{ }^{\alpha} r_{\alpha}+\frac{\left(\lambda \gamma^{m} \gamma^{n} \bar{\lambda}\right)}{2(\lambda \bar{\lambda})} \Pi_{m} \Gamma_{n}+\frac{\Gamma_{m} \Gamma_{n}}{4(\lambda \bar{\lambda})}\left[\left(\bar{\lambda} \gamma^{m n} \partial \theta\right)+\frac{\left(\lambda \gamma^{m n} \bar{\lambda}\right)}{(\lambda \bar{\lambda})}(\bar{\lambda} \partial \theta)\right]+c T-b c \partial c\right. \\
& +\widehat{\gamma}\left[b+\bar{\Gamma}^{m} \Pi_{m}+\frac{\bar{\Gamma}_{m} \bar{\Gamma}_{n}}{4(\lambda \bar{\lambda})}\left(\lambda \gamma^{m n} r\right)+s^{\alpha} \partial \bar{\lambda}_{\alpha}+\frac{\left(\bar{\lambda} \gamma^{m n} \partial \theta\right)}{4(\lambda \bar{\lambda})} N_{m n}^{\prime}+\frac{(\bar{\lambda} \partial \theta)}{4(\lambda \bar{\lambda})}\left(J^{\prime}+4 u_{n} v^{n}\right)+\left(\lambda \gamma^{m} \partial \theta\right) v_{m}\right] \\
& \left.+u_{m}\left[\bar{\Gamma}^{m}+\frac{1}{2(\lambda \bar{\lambda})} \bar{\lambda} \gamma^{m} d-\frac{\left(\bar{\lambda} \gamma^{m} \gamma^{n p} r\right)}{8(\lambda \bar{\lambda})^{2}}\left(N_{n p}^{\prime}+\frac{1}{\widehat{\gamma}} \Gamma_{n} \Gamma_{p}\right)\right]\right)
\end{aligned}
$$

where $N_{m n}^{\prime}=\frac{1}{2}\left(\lambda \gamma_{m n} w^{\prime}\right), J^{\prime}=-\lambda^{\alpha} w_{\alpha}^{\prime}$, and

$$
\widetilde{\bar{w}}^{\alpha} \equiv \bar{w}^{\alpha}+\frac{1}{2(\lambda \bar{\lambda})}\left(\lambda \gamma_{m} \gamma_{n}\right)^{\alpha}\left(u_{m} v_{n}-\Gamma_{m} \bar{\Gamma}_{n}\right)
$$

Since $\widetilde{\bar{w}}^{\alpha}$ of (2.38) commutes with the constraints $v^{m}\left(\gamma_{m} \bar{\lambda}\right)^{\alpha}=0$ and $\bar{\Gamma}^{m}\left(\gamma_{m} \bar{\lambda}\right)^{\alpha}=0$ of (2.21) and (2.32) up to the gauge transformation $\delta{\overline{\bar{w}}^{\prime}}^{\alpha}=f^{m}\left(\bar{\lambda} \gamma_{m}\right)^{\alpha}$, one can easily verify that $Q$ of (2.37) also commutes with these constraints.

The BRST operator of (2.37) is closely related to the simplified form of the composite pure spinor $b$ ghost found in [17]. The third line of (2.37) is $u_{m}$ times the constraint in [17] for $\bar{\Gamma}^{m}$, and the second line of (2.37) contains $\widehat{\gamma}$ times the composite $b$ ghost expressed in terms of $\bar{\Gamma}^{m}$. After applying the similarity transformation $\mathcal{O} \rightarrow e^{-S} e^{-R} \mathcal{O} e^{R} e^{S}$ where

$$
\begin{aligned}
& R=\int d z \frac{1}{2(\lambda \bar{\lambda})} \Gamma^{m}\left[\bar{\lambda} \gamma^{m} d-\frac{\left(\bar{\lambda} \gamma^{m} \gamma^{n p} r\right)}{4(\lambda \bar{\lambda})}\left(N_{n p}^{\prime}+\frac{1}{\widehat{\gamma}} \Gamma_{n} \Gamma_{p}\right)\right], \\
& S=-\int d z \widehat{\gamma} v^{m}\left(\Pi_{m}+\frac{\left(\lambda \gamma^{m} \gamma^{n} r\right)}{4(\lambda \bar{\lambda})} \bar{\Gamma}_{n}\right)
\end{aligned}
$$

(2.37) reduces to

$$
Q=\int d z\left(\lambda^{\alpha} d_{\alpha}+\widetilde{\bar{w}}^{\alpha} r_{\alpha}+\widehat{\gamma}\left[b-B+v_{m}\left(\lambda \gamma^{m}\right)_{\alpha} \partial\left(\frac{\Gamma_{n}\left(\gamma^{n} \bar{\lambda}\right)^{\alpha}}{2(\lambda \bar{\lambda})}\right)\right]+u_{m} \bar{\Gamma}^{m}+c T-b c \partial c\right)
$$


where $B$ is the usual composite pure spinor $b$ ghost (ignoring normal ordering terms)

$$
\begin{aligned}
B & =-s^{\alpha} \partial \bar{\lambda}_{\alpha}+\frac{\bar{\lambda}_{\alpha}\left(2 \Pi^{m}\left(\gamma_{m} d\right)^{\alpha}-N_{m n}^{\prime}\left(\gamma^{m n} \partial \theta\right)^{\alpha}-J^{\prime} \partial \theta^{\alpha}\right)}{4(\bar{\lambda} \lambda)} \\
& -\frac{\left(\bar{\lambda} \gamma^{m n p} r\right)\left(d \gamma_{m n p} d+24 N_{m n}^{\prime} \Pi_{p}\right)}{192(\bar{\lambda} \lambda)^{2}}+\frac{\left(r \gamma_{m n p} r\right)\left(\bar{\lambda} \gamma^{m} d\right) N^{\prime n p}}{16(\bar{\lambda} \lambda)^{3}}-\frac{\left(r \gamma_{m n p} r\right)\left(\bar{\lambda} \gamma^{p q r} r\right) N^{\prime m n} N_{q r}^{\prime}}{128(\bar{\lambda} \lambda)^{4}} .
\end{aligned}
$$

Finally, the similarity transformation $\mathcal{O} \rightarrow e^{-U} \mathcal{O} e^{U}$ where

$$
U=\int d z c\left(B-v_{m}\left(\lambda \gamma^{m}\right)_{\alpha} \partial\left(\frac{\Gamma_{n}\left(\gamma^{n} \bar{\lambda}\right)^{\alpha}}{2(\lambda \bar{\lambda})}\right)-\widehat{\beta} \partial c\right)
$$

transforms (2.40) into

$$
Q=\int d z\left(\lambda^{\alpha} d_{\alpha}+\widetilde{\bar{w}}^{\prime} r_{\alpha}+\widehat{\gamma} b+u_{m} \bar{\Gamma}^{m}\right)
$$

Note that this last similarity transformation shifts the Virasoro $b$ ghost to

$$
e^{-U} b e^{U}=b+B-v_{m}\left(\lambda \gamma^{m}\right)_{\alpha} \partial\left(\frac{\Gamma_{n}\left(\gamma^{n} \bar{\lambda}\right)^{\alpha}}{2(\lambda \bar{\lambda})}\right)-\widehat{\beta} \partial c-\partial(\widehat{\beta} c)
$$

The usual quartet argument implies that the cohomology of (2.43) is independent of $\left(u_{m}, v^{m}\right),\left(\Gamma_{m}, \bar{\Gamma}^{m}\right),(\widehat{\gamma}, \widehat{\beta}),(b, c),\left(\bar{\lambda}_{\alpha}, \widetilde{\widetilde{w}}^{\alpha}\right)$, and $\left(r_{\alpha}, s^{\alpha}\right)$, so one recovers the original pure spinor BRST operator $Q_{\text {pure }}=\int d z \lambda^{\alpha} d_{\alpha}$.

So after adding non-minimal spinors and twisting the spin-half $\psi^{m}$ variables into spinzero and spin-one variables, the RNS BRST operator has been covariantly mapped into the pure spinor BRST operator. In the next two sections, this covariant map will be used to relate vertex operators and scattering amplitudes in the two formalisms.

\section{$3 \quad$ Vertex operators}

In this section, massless vertex operators in the RNS and pure spinor formalisms will be related to each other using the covariant map of the previous section. After adding the non-minimal spinor variables of (2.5) to the RNS formalism, both Neveu-Schwarz and Ramond vertex operators can be constructed in the "zero picture" without spin fields or bosonization. In this non-minimal RNS formalism, vertex operators in the $G S O(+)$ sector can be expressed in $\mathrm{d}=10$ superspace and there is no difficulty with describing RamondRamond backgrounds. After dynamically twisting and gauge-fixing, these massless vertex operators in the non-minimal RNS formalism reduce to the massless vertex operators in the pure spinor formalism.

\subsection{Non-minimal RNS vertex operators}

After adding the non-minimal spinor variables of (2.5) and performing the similarity transformation of (2.12), the non-minimal RNS BRST operator of (2.13) takes the manifestly spacetime supersymmetric form

$$
Q=\int d z\left(\Lambda^{\alpha} d_{\alpha}+\frac{1}{2 \gamma}\left(\Lambda \gamma^{m} \Lambda\right) \psi_{m}+c T+\gamma \psi^{m} \Pi_{m}+\gamma^{2}\left(b+\Omega_{\alpha} \partial \theta^{\alpha}\right)-b c \partial c\right) .
$$


To construct massless open superstring vertex operators in the ghost-number one cohomology of $Q$, the first step will be to use the "minimal coupling" construction of Siegel [24] in which the operators $\left[d_{\alpha}, \Pi_{m}, \partial \theta^{\alpha}\right]$ in $Q$ are replaced by the $\mathrm{d}=10$ super-Yang-Mills superfields $\left[A_{\alpha}(x, \theta),-A_{m}(x, \theta),-W^{\alpha}(x, \theta)\right]$. These superfields satisfy the on-shell constraints $[25,26]$

$$
\begin{aligned}
D_{\alpha} A_{\beta}+D_{\beta} A_{\alpha} & =\gamma_{\alpha \beta}^{m} A_{m}, \quad D_{\alpha} A_{m}-\partial_{m} A_{\alpha}=\left(\gamma_{m}\right)_{\alpha \beta} W^{\beta}, \\
D_{\alpha} W^{\beta} & =\frac{1}{2}\left(\gamma^{m n}\right)_{\alpha}^{\beta} \partial_{m} A_{n}=\frac{1}{4}\left(\gamma^{m n}\right)_{\alpha}{ }^{\beta} F_{m n},
\end{aligned}
$$

and are defined up to the gauge transformation

$$
\delta A_{\alpha}=D_{\alpha} \Sigma, \quad \delta A_{m}=\partial_{m} \Sigma
$$

where $D_{\alpha}=\frac{\partial}{\partial \theta^{\alpha}}+\frac{1}{2}\left(\gamma^{m} \theta\right)_{\alpha} \partial_{m}$ is the $\mathrm{d}=10$ supersymmetric derivative. In components, one can gauge

$$
\begin{aligned}
A_{\alpha} & =\frac{1}{2}\left(\gamma^{m} \theta\right)_{\alpha} a_{m}+\frac{1}{3}\left(\gamma^{m} \theta\right)_{\alpha}\left(\gamma_{m} \theta\right)_{\beta} \xi^{\beta}+\ldots, \quad A_{m}=a_{m}+\left(\gamma_{m} \theta\right)_{\alpha} \xi^{\alpha}+\ldots \\
W^{\alpha} & =\xi^{\alpha}-\frac{1}{2}\left(\gamma^{m n} \theta\right)^{\alpha} \partial_{m} a_{n}+\ldots, \quad F_{m n}=\partial_{m} a_{n}-\partial_{n} a_{m}+\ldots
\end{aligned}
$$

where $a_{m}(x)$ and $\xi^{\alpha}(x)$ are the onshell gluon and gluino fields satisfying $\partial^{m} \partial_{[m} a_{n]}=0$ and $\partial_{m}\left(\gamma^{m} \xi\right)_{\alpha}=0$, and $\ldots$ denotes terms higher-order in $\theta^{\alpha}$ which can be expressed in terms of derivatives of $a_{m}$ and $\xi^{\alpha}$.

So the "minimal coupling" construction of Siegel predicts the massless vertex operator

$$
\begin{aligned}
V_{\min }= & \Lambda^{\alpha} A_{\alpha}(x, \theta)-\gamma \psi^{m} A_{m}(x, \theta)-\gamma^{2} \Omega_{\alpha} W^{\alpha}(x, \theta) \\
& +c\left(\partial \theta^{\alpha} A_{\alpha}(x, \theta)+\Pi^{m} A_{m}(x, \theta)+d_{\alpha} W^{\alpha}(x, \theta)\right) .
\end{aligned}
$$

Using the constraints of (3.2), one finds that $Q V_{\min }$ is nonzero and satisfies

$$
Q V_{\min }=Q\left[\frac{1}{2} c\left(\psi^{m} \psi^{n}-\frac{1}{2} \Lambda \gamma^{m n} \Omega\right) F_{m n}(x, \theta)+c \gamma \psi^{m} \Omega_{\alpha} \partial_{m} W^{\alpha}(x, \theta)\right] .
$$

So the minimal coupling construction needs to be slightly corrected and the ghost-number one BRST-invariant vertex operator is

$$
\begin{aligned}
V= & \Lambda^{\alpha} A_{\alpha}(x, \theta)-\gamma \psi^{m} A_{m}(x, \theta)-\gamma^{2} \Omega_{\alpha} W^{\alpha}(x, \theta) \\
& +c\left(\partial \theta^{\alpha} A_{\alpha}(x, \theta)+\Pi^{m} A_{m}(x, \theta)+d_{\alpha} W^{\alpha}(x, \theta)\right) \\
& -\frac{1}{2} c\left(\psi^{m} \psi^{n}-\frac{1}{2} \Lambda \gamma^{m n} \Omega\right) F_{m n}(x, \theta)-c \gamma \psi^{m} \Omega_{\alpha} \partial_{m} W^{\alpha}(x, \theta) .
\end{aligned}
$$

The integrated BRST-invariant vertex operator of ghost-number zero is defined in the usual manner as $\int d z U \equiv\left\{\int d z b, V\right\}$, so the integrated vertex operator is

$$
\begin{aligned}
\int d z U= & \int d z\left[\partial \theta^{\alpha} A_{\alpha}(x, \theta)+\Pi^{m} A_{m}(x, \theta)+d_{\alpha} W^{\alpha}(x, \theta)\right. \\
& \left.+\frac{1}{2}\left(-\psi^{m} \psi^{n}+\frac{1}{2} \Lambda \gamma^{m n} \Omega\right) F_{m n}(x, \theta)-\gamma \psi^{m} \Omega_{\alpha} \partial_{m} W^{\alpha}(x, \theta)\right] .
\end{aligned}
$$


The term $\frac{1}{2}\left(-\psi^{m} \psi^{n}+\frac{1}{2} \Lambda \gamma^{m n} \Omega\right) F_{m n}(x, \theta)$ in (3.8) is expected since when $F_{m n}$ is constant, the integrated vertex operator should be the Lorentz generator

$$
\int d z\left[x^{[m} \partial x^{n]}-\psi^{m} \psi^{n}-\frac{1}{2}\left(\theta \gamma^{m n} p\right)+\frac{1}{2}\left(\Lambda \gamma^{m n} \Omega\right)\right]
$$

where the $x^{[m} \partial x^{n]}-\frac{1}{2}\left(\theta \gamma^{m n} p\right)$ contribution to (3.9) comes from the $\partial \theta^{\alpha} A_{\alpha}+\Pi^{m} A_{m}+d_{\alpha} W^{\alpha}$ terms in (3.8). However, the presence of the $-\gamma \psi^{m} \Omega_{\alpha} \partial_{m} W^{\alpha}(x, \theta)$ term in (3.8) is surprising and it would be useful to get a better understanding of this term.

By adding the integrated vertex operator of (3.8) to the open superstring worldsheet action of (2.5), one can describe super-Yang-Mills backgrounds with both Neveu-Schwarz and Ramond background fields turned on. And by taking the "left-right product" of two open superstring vertex operators and adding to the closed superstring worldsheet action, one can describe $\mathrm{d}=10$ supergravity backgrounds in the non-minimal RNS formalism which include Ramond-Ramond background fields.

Despite the $\frac{1}{\gamma}$ dependence in the similarity transformation of (2.12) and in the BRST operator of (2.13), the massless super-Yang-Mills vertex operator of (3.7) has no $\frac{1}{\gamma}$ dependence and is therefore in the "small" Hilbert space. And since all massive vertex operators in the $G S O(+)$ sector can be obtained from OPE's of the super-Yang-Mills vertex operators, all physical vertex operators in the $G S O(+)$ sector of the non-minimal RNS formalism can be constructed in the "small" Hilbert space.

On the other hand, the physical vertex operator for the Neveu-Schwarz tachyon in the non-minimal RNS formalism has $\frac{1}{\gamma}$ dependence and is

$$
V=e^{-R^{\prime}}\left[\left(\gamma+i c \psi^{m} k_{m}\right) e^{i k^{m} x_{m}}\right] e^{R^{\prime}}=\left(\gamma+i c\left(\psi^{m}-\frac{1}{2 \gamma} \Lambda \gamma^{m} \theta\right) k_{m}\right) e^{i k^{m} x_{m}}
$$

where $R^{\prime}$ is defined in (2.12). So it appears that vertex operators in the $G S O(-)$ sector of the non-minimal RNS formalism cannot be constructed in the "small" Hilbert space.

\subsection{Relation with RNS and pure spinor vertex operators}

To relate the non-minimal vertex operator of (3.7) with the minimal RNS vertex operator for the gluon, one gauges $\theta^{\alpha}=\Lambda^{\alpha}=0$ in (3.7) to obtain

$$
V=-\gamma \psi^{m} a_{m}(x)+c\left(\partial x^{m} a_{m}(x)-\psi^{m} \psi^{n} \partial_{m} a_{n}(x)\right)
$$

which is the standard RNS gluon vertex operator. However, because there are no spin fields in the non-minimal RNS vertex operators, it is unclear how to relate the non-minimal and minimal RNS vertex operators for the gluino.

To relate the non-minimal vertex operator of (3.7) with the super-Yang-Mills vertex operator in the pure spinor formalism, one gauges $u_{m}=\Gamma_{m}=c=\widehat{\gamma}=0$. In this gauge, $\Lambda^{\alpha}$ reduces to $\lambda^{\alpha}$ and the vertex operators of (3.7) and (3.8) reduce to

$$
\begin{aligned}
V & =\lambda^{\alpha} A_{\alpha}(x, \theta) \\
\int d z U & =\int d z\left[\partial \theta^{\alpha} A_{\alpha}(x, \theta)+\Pi^{m} A_{m}(x, \theta)+d_{\alpha} W^{\alpha}(x, \theta)+\frac{1}{4}\left(\lambda \gamma^{m n} w\right) F_{m n}(x, \theta)\right]
\end{aligned}
$$


which are the standard unintegrated and integrated super-Yang-Mills vertex operators in the pure spinor formalism.

So the vertex operator of (3.7) in the non-minimal RNS formalism provides a bridge between the usual RNS and pure spinor vertex operators. Surprisingly, the non-minimal vertex operators for Ramond states do not require spin fields or bosonization, and the non-minimal vertex operators for states in the $G S O(-)$ sector cannot be expressed in the "small" Hilbert space. It would be very useful to understand how to relate these nonminimal RNS vertex operators with the usual RNS vertex operators for Ramond states and $G S O(-)$ states.

\section{Scattering amplitudes}

In this section, dynamical twisting will be argued to transform the RNS amplitude prescription into the pure spinor amplitude prescription. So assuming that dynamical twisting is an allowable field redefinition at the quantum level, the RNS and pure spinor amplitude prescriptions are expected to be equivalent. However, it should be stressed that there are various subtleties with both the RNS and pure spinor amplitude prescriptions and the argument sketched here does not address these subtleties. For example, the non-split nature of super-moduli space in the RNS formalism [27-29] makes it difficult to compute multillop amplitudes using picture-changing operators. And in the pure spinor formalism, the presence in multiloop amplitudes of poles when $(\lambda \bar{\lambda}) \rightarrow 0$ requires special regulators [30] which complicate the computation of higher-genus terms that are not protected by supersymmetry.

In string theories with chiral bosons, functional integration over the chiral boson zero modes needs to be regularized. As long as the regularization method preserves BRST invariance, on-shell amplitudes are expected to be independent of the regularization method. A convenient BRST-invariant method for regularizing the functional integration over chiral bosons is to insert a picture-changing operator for each chiral boson zero mode. Dynamical twisting modifies the structure of the chiral bosons and therefore modifies the picturechanging operators used to regularize their functional integration. By taking into account this modification coming from dynamical twisting, the RNS amplitude prescription can be related to the pure spinor amplitude prescription.

\subsection{Non-minimal RNS amplitude prescription}

In the RNS formalism, the $(\beta, \gamma)$ chiral bosons carry conformal weight $\left(\frac{3}{2},-\frac{1}{2}\right)$ and therefore have $(0,2)$ zero modes on a genus zero surface, $(1,1)$ zero modes on a genus one surface, and $(2 g-2,0)$ zero modes on a genus $g$ surface for $g>1$. For each $\gamma$ zero mode, one needs to insert a "picture-lowering" operator [3]

$$
Y_{\gamma}=c \delta^{\prime}(\gamma)=c \partial \xi e^{-2 \phi}
$$

where $\gamma=\eta e^{\phi}$ and $\beta=\partial \xi e^{-\phi}$. And for each $\beta$ zero mode, one needs to insert a "pictureraising" operator

$$
Z_{\beta}=: \delta(\beta) Q(\beta):=\delta(\beta)\left(\partial x^{m} \psi_{m}+\ldots\right)
$$


In the non-minimal RNS formalism, one also has the $\left(\Lambda^{\alpha}, \Omega_{\alpha}\right)$ chiral bosons of conformal weight $(0,1)$ which have $(16,16 g)$ zero modes on a genus $g$ surface. Using the non-minimal BRST operator of (3.1), one needs to insert for each $\Lambda^{\alpha}$ zero mode the BRSTinvariant picture-lowering operator

$$
Y_{\Lambda^{\alpha}}=e^{-R} \delta\left(\Lambda^{\alpha}\right) \theta^{\alpha} e^{R}=\delta\left(\Lambda^{\alpha}\right) \theta^{\alpha}-\delta^{\prime}\left(\Lambda^{\alpha}\right) c \theta^{\alpha} \partial \theta^{\alpha}
$$

where $R$ is defined in (2.9). And one needs to insert for each $\Omega_{\alpha}$ zero mode the BRSTinvariant picture-raising operator

$$
Z_{\Omega_{\alpha}}=: \delta\left(\Omega_{\alpha}\right)\left[Q, \Omega_{\alpha}\right]:=\delta\left(\Omega_{\alpha}\right)\left(d_{\alpha}+\ldots\right) .
$$

For the scattering of external gluons, one can verify that the picture-lowering and picture-raising operators of (4.3) and (4.4) absorb all the zero modes of $\left(\Lambda^{\alpha}, \Omega_{\alpha}\right)$ and $\left(\theta^{\alpha}, p_{\alpha}\right)$. Furthermore, the functional integral over the bosonic non-zero modes of $\left(\Lambda^{\alpha}, \Omega_{\alpha}\right)$ cancels the functional integral over the fermionic non-zero modes of $\left(\theta^{\alpha}, p_{\alpha}\right)$. So after performing the functional integration over the $\left(\Lambda^{\alpha}, \Omega_{\alpha}\right)$ and $\left(\theta^{\alpha}, p_{\alpha}\right)$ variables, the amplitude prescription for external gluon scattering coincides with the usual RNS amplitude prescription.

However, for scattering involving external gluinos, the non-minimal RNS prescription is very different from the usual RNS prescription since the non-minimal Ramond vertex operators do not contain spin fields or half-integer picture. It would be fascinating to find a proof that scattering amplitudes involving external gluinos coincide in the non-minimal and minimal RNS formalisms.

\subsection{Pure spinor amplitude prescription}

To relate the non-minimal RNS formalism with the pure spinor formalism, one needs to dynamically twist the spin-half fermions $\psi^{m}$ into spin-zero and spin-one fermions $\Gamma_{m}$ and $\bar{\Gamma}^{m}$ using pure spinors $\left(\lambda^{\alpha}, \bar{\lambda}_{\alpha}\right)$ constructed from the unconstrained spinor $\Lambda^{\alpha}=$ $\lambda^{\alpha}+\frac{1}{2(\lambda \bar{\lambda})} u_{m}\left(\gamma^{m} \bar{\lambda}\right)^{\alpha}$ of (2.17). In addition, one needs to replace the $(\beta, \gamma)$ ghosts of conformal weight $\left(\frac{3}{2},-\frac{1}{2}\right)$ with $(\widehat{\beta}, \widehat{\gamma})$ ghosts of conformal weight $(2,-1)$ where $\widehat{\gamma} \equiv(\gamma)^{2}$. As will now be argued, the different zero mode structure of chiral bosons created by this dynamical twisting will modify the RNS scattering amplitude prescription into the pure spinor scattering amplitude prescription. So if dynamical twisting can be proven at the quantum level to be a consistent field redefinition, the scattering amplitude prescriptions in the two formalisms should be equivalent.

After dynamical twisting, the chiral bosons include the pure spinor variables $\left(\lambda^{\alpha}, w_{\alpha}\right)$ and $\left(\bar{\lambda}_{\alpha}, \bar{w}^{\alpha}\right)$ of conformal weight $(0,1)$, the $\left(u^{m}, v_{m}\right)$ variables of conformal weight $(0,1)$, and the $(\widehat{\beta}, \widehat{\gamma})$ variables of conformal weight $(2,-1)$. Functional integration over the zero modes of the pure spinors $\left(\lambda^{\alpha}, w_{\alpha}\right)$ and $\left(\bar{\lambda}_{\alpha}, \bar{w}^{\alpha}\right)$ can be performed using the standard pure spinor regulator [31]

$$
\mathcal{N}=e^{-\left\{Q, \theta^{\alpha} \bar{\lambda}_{\alpha}+\sum_{I=1}^{g} w_{\alpha I} s_{I}^{\alpha}\right\}}=e^{-\left(\lambda^{\alpha} \bar{\lambda}_{\alpha}+\theta^{\alpha} r_{\alpha}+\sum_{I=1}^{g}\left(w_{\alpha I} \bar{w}_{I}^{\alpha}+d_{\alpha I} s_{I}^{\alpha}\right)+\ldots\right)}
$$


where $\left(w_{\alpha I}, \bar{w}_{I}^{\alpha}, s_{I}^{\alpha}, d_{\alpha I}\right)$ for $I=1$ to $g$ are the $g$ holomorphic zero modes of $\left(w_{\alpha}, \bar{w}^{\alpha}, s^{\alpha}, d_{\alpha}\right)$ and

$$
Q=\int d z\left(\lambda^{\alpha} d_{\alpha}+\widetilde{\bar{w}}^{\alpha} r_{\alpha}+u_{m} \bar{\Gamma}^{m}+\widehat{\gamma}(b-B+\ldots)+c T-b c \partial c\right)
$$

is the BRST operator of (2.40). For each zero mode of $u_{m}$, one needs to insert the picturelowering operator

$$
Y_{u^{m}}=\delta\left(u_{m}\right) \Gamma_{m}
$$

And for each zero mode of $v_{m}$, one needs to insert the picture-raising operator

$$
Z_{v_{m}}=: \delta\left(v_{m}\right)\left[Q, v_{m}\right]:=\delta\left(v_{m}\right) \bar{\Gamma}_{m} .
$$

Finally, for each zero mode of $\widehat{\gamma}$, one needs to insert the picture-lowering operator

$$
Y_{\widehat{\gamma}}=\delta(\widehat{\gamma}) c
$$

And for each zero mode of $\widehat{\beta}$, one needs to insert the picture-raising operator

$$
Z_{\widehat{\beta}}=: \delta(\widehat{\beta})[Q, \widehat{\beta}]:=\delta(\widehat{\beta})(b-B+\ldots)
$$

where $B$ is the pure spinor $b$ ghost of $(2.41)$.

Since $(\widehat{\beta}, \widehat{\gamma})$ have the same conformal weight as the $(b, c)$ Virasoro ghosts, they have the same number of zero modes on the worldsheet. To reproduce the pure spinor amplitude prescription, the picture-lowering operators $Y_{\widehat{\gamma}}$ of (4.9) should be inserted on the unintegrated vertex operators and the picture-raising operators $Z_{\widehat{\beta}}$ of (4.10) should be inserted on the $(3 g-3) b$ ghosts contracted with the Beltrami differentials. With this choice, the contribution from each unintegrated vertex operator is $c \delta(\widehat{\gamma})\left(\lambda^{\alpha} A_{\alpha}(x, \theta)+\ldots\right)$ and the contribution from each of the $(3 g-3)$ Beltrami differentials is $b \delta(\widehat{\beta})(b-B+\ldots)$.

After inserting all the picture-lowering and picture-raising operators of (4.7)-(4.10), functional integration over the $\left(u_{m}, v^{m}\right)$ variables cancels the functional integration over the $\left(\Gamma_{m}, \bar{\Gamma}^{m}\right)$ variables and functional integration over the $(b, c)$ variables cancels the functional integration over the $(\widehat{\beta}, \widehat{\gamma})$ variables. The functional integral over the remaining variables with the regulator of (4.5) reproduces the usual pure spinor amplitude prescription where the $(3 g-3)$ Beltrami differentials are contracted with the $B$ operator of (2.41).

So after dynamically twisting and inserting the appropriate picture-lowering and picture-raising operators to regularize the functional integration over the chiral boson zero modes, the non-minimal RNS scattering amplitude prescription reduces to the usual pure spinor amplitude prescription.

But as was mentioned earlier, there are several subtleties which have been ignored in this argument. For example, the functional integral in the pure spinor formalism at higher genus is singular if there are poles of order $(\lambda \bar{\lambda})^{-11}$ coming from the $(3 g-3)$ pure spinor $B$ ghosts [30]. And in the RNS formalism, the non-split structure of higher genus supermoduli space [27-29] complicates the computation using picture-changing operators. It would be very interesting if these multiloop subtleties in the two formalisms could be related to each other using the covariant map of this paper. 


\section{Acknowledgments}

I would like to thank Nikita Nekrasov and Edward Witten for useful discussions, Sebastian Guttenberg for informing me about reference [18, 19], and CNPq grant 300256/94-9 and FAPESP grants 09/50639-2 and 11/11973-4 for partial financial support.

Open Access. This article is distributed under the terms of the Creative Commons Attribution License (CC-BY 4.0), which permits any use, distribution and reproduction in any medium, provided the original author(s) and source are credited.

\section{References}

[1] E. Witten, $D=10$ Superstring Theory, in proceedings of Grand Unification, Philadelphia, 1983, pg. 395.

[2] N. Berkovits, Quantization of the superstring with manifest $\mathrm{U}(5)$ superPoincaré invariance, Phys. Lett. B 457 (1999) 94 [hep-th/9902099] [INSPIRE].

[3] D. Friedan, E.J. Martinec and S.H. Shenker, Conformal Invariance, Supersymmetry and String Theory, Nucl. Phys. B 271 (1986) 93 [InSPIRE].

[4] N. Berkovits, Relating the RNS and pure spinor formalisms for the superstring, JHEP 08 (2001) 026 [hep-th/0104247] [INSPIRE].

[5] M. Tonin, World sheet supersymmetric formulations of Green-Schwarz superstrings, Phys. Lett. B 266 (1991) 312 [INSPIRE].

[6] M. Matone, L. Mazzucato, I. Oda, D. Sorokin and M. Tonin, The Superembedding origin of the Berkovits pure spinor covariant quantization of superstrings, Nucl. Phys. B 639 (2002) 182 [hep-th/0206104] [INSPIRE].

[7] D.P. Sorokin, Superbranes and superembeddings, Phys. Rept. 329 (2000) 1 [hep-th/9906142] [INSPIRE].

[8] N. Berkovits, Explaining the Pure Spinor Formalism for the Superstring, JHEP 01 (2008) 065 [arXiv: 0712.0324] [INSPIRE].

[9] N. Berkovits, Covariant quantization of the Green-Schwarz superstring in a Calabi-Yau background, Nucl. Phys. B 431 (1994) 258 [hep-th/9404162] [INSPIRE].

[10] L. Baulieu, Transmutation of pure $2-D$ supergravity into topological $2-D$ gravity and other conformal theories, Phys. Lett. B 288 (1992) 59 [hep-th/9206019] [INSPIRE].

[11] L. Baulieu, M.B. Green and E. Rabinovici, A Unifying topological action for heterotic and type-II superstring theories, Phys. Lett. B 386 (1996) 91 [hep-th/9606080] [InSPIRE].

[12] L. Baulieu, M.B. Green and E. Rabinovici, Superstrings from theories with $N>1$ world sheet supersymmetry, Nucl. Phys. B 498 (1997) 119 [hep-th/9611136] [INSPIRE].

[13] L. Baulieu and N. Ohta, World sheets with extended supersymmetry, Phys. Lett. B 391 (1997) 295 [hep-th/9609207] [INSPIRE].

[14] E. Witten, private communication.

[15] N. Nekrasov, Pure spinors, beta-gammas, super-Yang-Mills and Chern-Simons, Part 2, in KITP 2009 Lecture, http://online.kitp.ucsb.edu/online/strings09/nekrasov2/. 
[16] L. Baulieu, SU(5)-invariant decomposition of ten-dimensional Yang-Mills supersymmetry, Phys. Lett. B 698 (2011) 63 [arXiv:1009.3893] [INSPIRE].

[17] N. Berkovits, Dynamical twisting and the $b$ ghost in the pure spinor formalism, JHEP 06 (2013) 091 [arXiv: 1305.0693] [INSPIRE].

[18] P.A. Grassi and S. Guttenberg, On Projections to the Pure Spinor Space, JHEP 12 (2011) 089 [arXiv: 1109.2848] [INSPIRE].

[19] S. Guttenberg, A Projection to the Pure Spinor Space, arXiv:1202.0335 [INSPIRE].

[20] P.A. Grassi, G. Policastro, M. Porrati and P. Van Nieuwenhuizen, Covariant quantization of superstrings without pure spinor constraints, JHEP 10 (2002) 054 [hep-th/0112162] [INSPIRE].

[21] P.A. Grassi, G. Policastro and P. van Nieuwenhuizen, On the BRST cohomology of superstrings with/without pure spinors, Adv. Theor. Math. Phys. 7 (2003) 499 [hep-th/0206216] [INSPIRE].

[22] Y. Aisaka and Y. Kazama, A New first class algebra, homological perturbation and extension of pure spinor formalism for superstring, JHEP 02 (2003) 017 [hep-th/0212316] [INSPIRE].

[23] Y. Aisaka and Y. Kazama, Operator mapping between RNS and extended pure spinor formalisms for superstring, JHEP 08 (2003) 047 [hep-th/0305221] [INSPIRE].

[24] W. Siegel, Classical Superstring Mechanics, Nucl. Phys. B 263 (1986) 93 [InSPIRE].

[25] W. Siegel, Superfields in Higher Dimensional Space-time, Phys. Lett. B 80 (1979) 220 [INSPIRE].

[26] E. Witten, Twistor-Like Transform in Ten-Dimensions, Nucl. Phys. B 266 (1986) 245 [INSPIRE].

[27] R. Donagi and E. Witten, Supermoduli Space Is Not Projected, arXiv:1304.7798 [INSPIRE].

[28] E. Witten, More On Superstring Perturbation Theory, arXiv:1304.2832 [INSPIRE].

[29] E. Witten, Superstring Perturbation Theory Revisited, arXiv:1209.5461 [INSPIRE].

[30] N. Berkovits and N. Nekrasov, Multiloop superstring amplitudes from non-minimal pure spinor formalism, JHEP 12 (2006) 029 [hep-th/0609012] [INSPIRE].

[31] N. Berkovits, Pure spinor formalism as an $N=2$ topological string, JHEP 10 (2005) 089 [hep-th/0509120] [INSPIRE]. 